September 1993

McGill/93-46

\title{
Neutrino oscillations and the exact parity model
}

\author{
R. Foot \\ Physics Department, McGill University, 3600 University street, \\ Montreal, Quebec, Canada, Н3A 2T8
}

\begin{abstract}
We re-examine neutrino oscillations in exact parity models. Previously it was shown in a specific model that large neutrino mixing angles result. We show here that this is a general result of neutrino mixing in exact parity models provided that the neutrino mass matrix is real. In this case, the effects of neutrino mixing in exact parity models is such that the probability of a given weak eigenstate remaining in that eigenstate averages to less than a half when averaged over many oscillations. This result is interesting in view of the accumulating evidence for a significant deficit in the number of solar neutrinos. It may also be of relevance to the atmospheric neutrino anomaly.
\end{abstract}




\section{Introduction}

Neutrino physics is one field of research which may provide the first indication of physics beyond the minimal standard model. Indeed, there are already two exciting indications that the minimal standard model is incomplete. Firstly, there are the solar neutrino experiments [1]. For a long time there has been a discrepancy between the experimental measurements of Davis and the theoretical predictions. There are now four experiments which we summarise below:

$$
\begin{array}{ll}
\text { Homestake } & 0.27 \pm 0.04(\text { exp }) \pm 0.03(\text { theor }), \\
\text { Kamiokande } & 0.50 \pm 0.05(\text { stat }) \pm 0.06(\text { sys }) \pm 0.07(\text { theor }), \\
\text { Gallex } & 0.66 \pm 0.11(\text { stat }) \pm 0.05(\text { sys }) \pm 0.03(\text { theor }), \\
\text { SAGE } & 0.44_{-0.18}^{+0.13}(\text { stat }) \pm 0.11(\text { sys }) \pm 0.02(\text { theor }),
\end{array}
$$

where the data has been normalized to the theoretical prediction of Bachall et al [2]. Note that the theoretical prediction involves a lot of assumptions, and the true theoretical value may be outside these errors. In particular an analysis by S. Turck-Chieze et al [3] gives theoretical predictions for the experiements which are quite different to those of Bahcall but which still seem to be to high to be consistent with the data (although it has been argued that the data and the theory may be in-agreement if one takes into account all sources of uncertainty [4]). In view of the above, we do not attempt to propose a particular physics solution which will make all of the experiments agree with the theoretical prediction of Bahcall et al [2]. If there is a solar neutrino problem and if new particle physics is the solution then any new particle physics which can reduce the number of solar neutrinos by a large fraction (e.g. 1/2) may be the cause of the apparent disagreement of theory with data.

Another experiment which seems to be inconflict with theory is the atmospheric neutrino experiment [5]. This experiment measures the ratio of $\nu_{\mu} / \nu_{e}$ interactions where the neutrinos are presumed to originate from cosmic ray interactions in the atmosphere. These experiments observe a deficit in the ratio of $\nu_{\mu} / \nu_{e}$ interactions when the data is compared with theory. We summarise the situation below:

$$
\begin{array}{ll}
\text { Kamiokande } & 0.60 \pm 0.07(\text { stat }) \pm 0.05(\text { sys }), \\
I M B & 0.55 \pm 0.05(\text { stat }) \pm 0.10(\text { sys }),
\end{array}
$$


where the data has been normalized to the theoretically expected ratio [6].

One obvious way to try and reconcile these experiements with the standard model is to extend the model to incorporate neutrino masses [7]. Once neutrinos have masses, then oscillations of neutrino flavours can occur [7]. However, the effect of vaccum oscillations tends to be small unless there are large mixing angles. If neutrino masses follow the pattern suggested by the quarks, then mixing is small, and it is not possible to understand the solar neutrino (or atmospheric neutrino anomaly) in terms of vacuum oscillations. Neutrinos coming from the sun travel through matter and it is possible that the neutrino oscillations are matter enhanced (MSW effect [8]). This solution to the solar neutrino problem is probably the most economical solution in the sense that it proposes the least amount of new physics. This does not necessarily mean it is the correct solution (although it may be). Also, one cannot use the MSW effect to explain the atmospheric neutrino anomaly. If this anomaly is a signal for new partilce physics, then the most natural explanation for it is the existence of vacuum neutrino oscillations. It is possible that vacuum neutrino oscillations is also the physical mechanism responsible for the solar neutrino deficit. This is what we assume in this note. One of the theoretical problems with this interpretation is that it is hard to understand why the neutrino oscillations are so big?

Recently, it was pointed out [9] that if the minimal standard model is extended so that an exact discrete Parity symmetry exists $[9,10,11]$ (which is unbroken by the vaccuum), then in that framework it is quite natural for neutrino flavours to be maximally mixed combinations of ordinary and mirror matter. Mirror matter is essentially sterile (when probed with ordinary matter) so that if the oscillation lengths are small enough, then the vacuum neutrino oscillations will reduce the neutrino flux by a factor of two. In the previous work [9], the considerations were restricted to a specific model. Here we show that if the neutrino mass matrix is real, then in general, vacuum neutrino oscillations will deplete a weak eigenstate by at least a factor of two. This is an interesting result in view of the solar neutrino and atmospheric neutrino experiments.

The outline of this paper is as follows: In section II we briefly review the exact parity model. In section III we show that if neutrinos have mass and the ordinary and mirror neutrinos mix together, then in general the oscillations will be large. In fact, we show that the effects of neutrino mixing in exact parity models is such that the probability of a given weak eigenstate 
remains in that eigenstate is less than $1 / 2$ when it is averaged over many oscillations (provided that the neutrino mass matrix is real). In section IV we look at some examples. We examine some exact parity versions of the see-saw model. In section $\mathrm{V}$ we conclude with some comments.

\section{Exact parity models}

In this section we quickly review exact parity symmetric models $[9,10,11]$. To understand how parity might be conserved, consider a model which successfully describes present experiments. In particular, consider the minimal standard model. This model is described by a Lagrangian $\mathcal{L}_{1}$. This Lagrangian is not invariant under the usual parity transformation so it seems parity is violated. However, this Lagrangian may not be complete. If we add to $\mathcal{L}_{1}$ a new Lagrangian $\mathcal{L}_{2}$ which is just like $\mathcal{L}_{1}$ except that all left-handed (right-handed) fermions are replaced by new right-handed (left-handed) fermions which feel new interactions of the same form and strength, then the theory described by $\mathcal{L}=\mathcal{L}_{1}+\mathcal{L}_{2}$ is invariant under a parity symmetry (under this symmetry $\mathcal{L}_{1} \leftrightarrow \mathcal{L}_{2}$ ). In addition to these Lagrangian terms, there may also be terms which mix ordinary with mirror matter and which are parity invariant. We label this part of the Lagrangian as $\mathcal{L}_{\text {int }}$. The terms

in $\mathcal{L}_{\text {int }}$ are very important since they lead to interactions between ordinary and mirror matter, and hence allow the idea to be experimentally tested in the laboratory.

If we apply the above proceedure to the standard model then $\mathcal{L}_{1}$ is just the standard model Lagrangian. We now add the "mirror matter" as described above, so that the total Lagrangian consists of two parts $\mathcal{L}_{1}$ and $\mathcal{L}_{2}$. Then the gauge symmetry of the theory is

$$
S U(3)_{1} \otimes S U(2)_{1} \otimes U(1)_{1} \otimes S U(3)_{2} \otimes S U(2)_{2} \otimes U(1)_{2}
$$

There are two sets of fermions, the ordinary particles and their mirror images, 
which transform under the gauge group of Eq.(3) as

$$
\begin{array}{lll}
f_{L} \sim(1,2,-1)(1,1,0), & & F_{R} \sim(1,1,0)(1,2,-1), \\
e_{R} \sim(1,1,-2)(1,1,0), & & E_{L} \sim(1,1,0)(1,1,-2), \\
q_{L} \sim(3,2,1 / 3)(1,1,0), & & Q_{R} \sim(1,1,0)(3,2,1 / 3), \\
u_{R} \sim(3,1,4 / 3)(1,1,0), & & U_{L} \sim(1,1,0)(3,1,4 / 3), \\
d_{R} \sim(3,1,-2 / 3)(1,1,0), & D_{L} \sim(1,1,0)(3,1,-2 / 3),
\end{array}
$$

(with generation index suppressed). The Lagrangian is invariant under the discrete $Z_{2}$ parity symmetry defined by

$$
\begin{gathered}
x \rightarrow-x, t \rightarrow t, \\
G_{1}^{\mu} \leftrightarrow G_{2 \mu}, W_{1}^{\mu} \leftrightarrow W_{2 \mu}, B_{1}^{\mu} \leftrightarrow B_{2 \mu}, \\
f_{L} \leftrightarrow \gamma_{0} F_{R}, e_{R} \leftrightarrow \gamma_{0} E_{L}, q_{L} \leftrightarrow \gamma_{0} Q_{R}, u_{R} \leftrightarrow \gamma_{0} U_{L}, d_{R} \leftrightarrow \gamma_{0} D_{L},
\end{gathered}
$$

where $G_{1}^{\mu}\left(G_{2}^{\mu}\right), W_{1}^{\mu}\left(W_{2}^{\mu}\right)$ and $B_{1}^{\mu}\left(B_{2}^{\mu}\right)$ are the gauge bosons of the $S U(3)_{1}$ $\left(S U(3)_{2}\right), S U(2)_{1}\left(S U(2)_{2}\right), U(1)_{1}\left(U(1)_{2}\right)$ gauge forces respectively. The minimal model contains two Higgs doublets which are also parity partners:

$$
\phi_{1} \sim(1,2,1)(1,1,0), \phi_{2} \sim(1,1,0)(1,2,1) .
$$

An important feature which distinguishes this parity conserving theory from other such theories (e.g. the usual left-right symmetric model) is that the parity symmetry is assumed to be unbroken by the vacuum. The most general renormalizable Higgs potential can be written in the form

$$
V\left(\phi_{1}, \phi_{2}\right)=\lambda_{1}\left(\phi_{1}^{\dagger} \phi_{1}+\phi_{2}^{\dagger} \phi_{2}-2 u^{2}\right)^{2}+\lambda_{2}\left(\phi_{1}^{\dagger} \phi_{1}-\phi_{2}^{\dagger} \phi_{2}\right)^{2}
$$

where $\lambda_{1,2}$ and $u$ are arbitrary constants. In the region of parameter space where $\lambda_{1,2}>0, V\left(\phi_{1}, \phi_{2}\right)$ is non-negative and is minimized by the vacuum

$$
\left\langle\phi_{1}\right\rangle=\left\langle\phi_{2}\right\rangle=\left(\begin{array}{l}
0 \\
u
\end{array}\right) .
$$

The vacuum values of both Higgs fields are exactly the same and hence parity is not broken by the vacuum in this theory.

If the solar system is dominated by the usual particles, then the theory agrees with present experiments. The idea can be tested in the laboratory because it is possible for the two sectors to interact with each other via $\mathcal{L}_{\text {int }}$. 
In the simplest case that we are considering at the moment (where $\mathcal{L}_{1}$ is the minimal standard model lagrangian), there are just two possible terms (i.e. gauge invariant and renormalizable) in $\mathcal{L}_{\text {int }}$. They are,

(1) The Higgs potential terms $\lambda \phi_{1}^{\dagger} \phi_{1} \phi_{2}^{\dagger} \phi_{2}$ in Eq.(7) and

(2) The gauge boson kinetic mixing term $\alpha F_{\mu \nu}^{1} F^{2 \mu \nu}$ where $F_{\mu \nu}^{1,2}=\partial_{\mu} B_{\nu}^{1,2}-$ $\partial_{\nu} B_{\mu}^{1,2}$.

The principle phenomenological effect of the term in (1) is to modify the interactions of the Higgs boson. This effect will be tested if or when the Higgs scalar is discovered. The details have been discussed in Ref.[9]. The principle phenomenological effect of the kinetic mixing term in (2) is to give small electric charges to the mirror partners of the ordinary charged fermions. This effect has also been discussed previously $[10,11]$.

\section{General considerations}

In the past there has been much speculation that neutrinos are massive. If this is the case then this will lead to another signature of exact parity symmetric gauge models. This is because $\mathcal{L}_{\text {int }}$ can contain neutrino mass terms which mix the ordinary and mirror matter. (Note that if electric charge is conserved, then it is not possible for $\mathcal{L}_{\text {int }}$ to contain mass terms mixing the charged fermions of ordinary matter with mirror matter, however neutrinos may be neutral so such mass terms are possible providing that the neutrinos have masses). The actual number of neutral Weyl fermions is not known, (although the number of weakly interacting light $S U(2)_{L}$ doublet neutrinos has been determined to be 3 by the LEP experiments) so we will start with some general results.

Suppose there are $n$ ordinary neutrino Weyl fields, and $n$ mirror neutrino Weyl fields

$$
\begin{gathered}
\nu_{1 L}, \nu_{2 L}, \ldots, \nu_{n L}, \\
N_{1 R}, N_{2 R}, \ldots, N_{n R},
\end{gathered}
$$

\footnotetext{
${ }^{1}$ Note that apriori it is possible to have parity diagonal neutrino fields (i.e. with the parity transformation $\left.N_{R} \leftrightarrow \gamma_{0}\left(N_{R}\right)^{c}\right)$. If these fields exist then the following arguement is still valid and Eq.(11) would still be true in this case.
} 
where $\nu_{i L} \leftrightarrow \gamma_{0} N_{i R}(i=1, \ldots, n)$ under the parity symmetry (Eq.(5)). The physics becomes clearer if we work in the parity diagonal basis,

$$
\nu_{i L}^{ \pm}=\frac{\nu_{i L} \pm\left(N_{i R}\right)^{c}}{\sqrt{2}} .
$$

Then, under parity, $\nu_{i L}^{ \pm} \rightarrow \pm \gamma_{0}\left(\nu_{i L}^{ \pm}\right)^{c}$. Observe that mass terms of the form $m_{i j} \bar{\nu}_{i L}^{+}\left(\nu_{j L}^{+}\right)^{c}+$ H.c. and $m_{i j}^{\prime} \bar{\nu}_{i L}^{-}\left(\nu_{j L}^{-}\right)^{c}+H . c$. are invariant under parity if $m_{i j}$ and $m_{i j}^{\prime}$ are real, while terms of the form $m_{i j}^{\prime \prime} \bar{\nu}_{i L}^{+}\left(\nu_{j L}^{-}\right)^{c}+H . c$. are parity odd if $m_{i j}^{\prime \prime}$ is real, and hence they are not invariant under the discrete symmetry (provided $m_{i j}^{\prime \prime}$ are real). Under this reality assumption, it follows that the $\nu^{+}$fields do not mix with the $\nu^{-}$fields. Thus every mass eigenstate neutrino field will be linear combinations of $\nu^{+}$fields only, or linear combinations of $\nu^{-}$fields only. From this result one can easily show that an ordinary weak eigenstate such as the left-handed neutrino field coupling to the left-handed electron and the $W$ boson will have the form:

$$
\begin{gathered}
\nu_{e L}=\frac{\nu_{1}^{+}+\nu_{1}^{-}}{\sqrt{2}}, \\
=\sum_{i=1}^{n} \frac{\alpha_{i} \nu_{i}^{\prime}}{\sqrt{2}}+\frac{\beta_{i} \nu_{i}^{\prime \prime}}{\sqrt{2}},
\end{gathered}
$$

where $\nu_{i}^{\prime}\left(\nu_{i}^{\prime \prime}\right)$ are the set of mass eigenstates which are mixtures of the $\nu_{i}^{+}\left(\nu_{i}^{-}\right)$ fields and $\alpha_{i}$, and $\beta_{i}$, are parameters which depend on the details of the neutrino mass matrix, but must satisfy $\sum_{i=1}^{n} \alpha_{i}^{2}=\sum_{i=1}^{n} \beta_{i}^{2}=1$.

Define $\mathcal{P}(E, R)$ to be the probability that an initial weak eigenstate electron neutrino (with energy $E$ ) remains a weak eigenstate neutrino after travelling a distance $R$. Then:

$$
\mathcal{P}(E, R)=\left|\left\langle\nu_{e L} \mid \nu_{e L}(E, R)\right\rangle\right|^{2} .
$$

Define $\mathcal{P}$ to be the averaged probability:

$$
\mathcal{P}=\frac{\text { limit }}{R \rightarrow \infty} \frac{1}{R} \int_{0}^{R} \mathcal{P}(E, R) d R .
$$

Calculating $\mathcal{P}$ we find $[$ :

$$
\mathcal{P}=\sum_{i=1}^{n} \frac{\alpha_{i}^{4}}{4}+\frac{\beta_{i}^{4}}{4} .
$$

\footnotetext{
${ }^{2}$ Note that we have assumed that there are no degeneracies among the masses of the physical neutrinos.
} 
Hence

$$
\frac{1}{2 n} \leq \mathcal{P} \leq \frac{1}{2}
$$

In the case of the solar neutrino problem, one must average $\mathcal{P}(E, R)$ by taking into account the region of emission of the sun, the region of absorption on Earth, and the energy spectrum of the source. In general such an average will depend on the mass differences of the physical neutrinos. However if the oscillation lengths are small enough then the average probability $\mathcal{P}$ (defined in Eq.(13)) will be a good aproximation to the fraction of electron neutrinos reaching the Earth. In particular, for the case of 2 state mixing, the averaged function $\mathcal{P}$ is $1 / 2$. In this simple case, for the neutrino energies detected by the solar neutrino experiments, the neutrino flux will be reduced by the factor $\mathcal{P}=1 / 2$ provided that the neutrino mass squared difference satisfies $[2]$

$$
0.014 e V^{2}>\Delta\left(m^{2}\right)>3 \times 10^{-10} e V^{2} .
$$

Note that the upper bound $\left(0.014 \mathrm{eV}^{2}\right)$ comes from laboratory experiments [12]. Observe that Eq.(16) represents a very large range of parameters (8 orders of magnitude). Thus there is actually a very large range of parameters in which the flux of electron neutrinos coming from the sun will be significantly reduced by a factor of 2 .

\section{Some examples: The see saw model.}

As an example, consider the exact parity version of the see-saw model. The see-saw model is a simple way to understand the smallness of the masses of the known (i.e. the three left-handed) neutrinos. We will actually consider two different versions of the see-saw model to illustrate the results of the last section. The first version of the see-saw model that we will use is the one with an additional right-handed gauge singlet neutrino (together with its mirror image) per generation. Before we consider the parity invariant case, we first define some notation by quickly reviewing the ordinary parity violating case. In this case there are two Weyl neutrino fields per generation. Denote these by $\nu_{L}$ and $\nu_{R}$. The $\nu_{L}$ field is a member of a $S U(2)_{L}$ doublet while $\nu_{R}$ is a gauge singlet. The usual Higgs doublet can couple the $\nu_{L}$ and $\nu_{R}$ together and its vacuum expectation value will generate a Dirac mass term. Also, 
since we assume that $\nu_{R}$ is electrically neutral it can have a bare Majorana mass term coupling it to itself. Thus we have two mass terms:

$$
\mathcal{L}_{\text {mass }}=2 m \bar{\nu}_{L} \nu_{R}+M \bar{\nu}_{R}\left(\nu_{R}\right)^{c}+H . c . .
$$

Note that $M$ is a bare mass term, and can take any value, while $m$ is a mass term which is generated when the electroweak gauge symmetry is broken. It is usually assumed that $M \gg m$. The mass matrix has the form:

$$
\mathcal{L}_{\text {mass }}=\left(\bar{\nu}_{L},\left(\bar{\nu}_{R}\right)^{c}\right)\left(\begin{array}{cc}
0 & m \\
m & M
\end{array}\right)\left(\begin{array}{c}
\left(\nu_{L}\right)^{c} \\
\nu_{R}
\end{array}\right)+H . c . .
$$

Diagonalising this mass matrix yields two Majorana mass eigenstates with masses $m^{2} / M$ and $M$ (assuming that $M \gg m$ ). If we denote the mass eigenstates by $\nu_{\text {light }}$ and $\nu_{\text {heavy }}$ then they can be written in terms of the weak eigenstates as follows:

$$
\begin{gathered}
\nu_{\text {light } L}=\cos \phi \nu_{L}+\sin \phi\left(\nu_{R}\right)^{c}, \\
\nu_{\text {heavy } R}=-\sin \phi\left(\nu_{L}\right)^{c}+\cos \phi \nu_{R},
\end{gathered}
$$

where $\tan \phi=m / M$. Thus in the limit $M \gg m$, we see that the light state is essentially $\nu_{L}$ while the heavy state is essentially $\nu_{R}$.

The see-saw model is a simple extension of the standard model. As in the case of the standard model, it is straightforward to make it exactly parity invariant. In this case, there are four Weyl neutrino fields per generation: $\nu_{L}, \nu_{R}$ and their mirror images $N_{R}, N_{L}$. Under the parity symmetry $\nu_{L, R} \leftrightarrow$ $\gamma_{0} N_{R, L}$. Note that since $N_{R}$ is the parity partner of $\nu_{L}$ it belongs to a $S U(2)_{2}$ doublet; while $N_{L}$ being the parity partner of $\nu_{R}$ is a gauge singlet. (Recall that the gauge group is defined in Eq.(3) and the parity transformations are given in Eq.(5)). Assuming the minimal Higgs sector of one ordinary Higgs doublet and its mirror image, then the following mass terms are allowed (where for simplicity we examine only one generation):

$$
\begin{aligned}
& \mathcal{L}_{\text {mass }}=2 m_{1}\left(\bar{\nu}_{L} \nu_{R}+\bar{N}_{R} N_{L}\right)+2 m_{2}\left(\bar{\nu}_{L}\left(N_{L}\right)^{c}+\bar{N}_{R}\left(\nu_{R}\right)^{c}\right) \\
& +M_{1}\left(\bar{\nu}_{R}\left(\nu_{R}\right)^{c}+\bar{N}_{L}\left(N_{L}\right)^{c}\right)+M_{2}\left(\bar{\nu}_{R} N_{L}+\bar{N}_{L} \nu_{R}\right)+H . c . .
\end{aligned}
$$

Note that $m_{1,2}$ are mass terms which arise from spontaneous symmetry breaking, while $M_{1,2}$ are bare mass terms. As in the case discussed above, we will 
assume that $M_{1,2} \gg m_{1,2}$. We will also assume that the masses are real. This is an important restriction and it is essentially the only way in which we depart from the most general case. From Eq.(20) we see that the mass matrix has the form:

$$
\mathcal{L}_{\text {mass }}=\bar{\nu}_{L} \mathcal{M}\left(\nu_{L}\right)^{c}+\text { H.c. },
$$

where

$$
\nu_{L}=\left(\left(\nu_{L}\right)^{c}, N_{R}, \nu_{R},\left(N_{L}\right)^{c}\right)^{T}
$$

and

$$
\mathcal{M}=\left(\begin{array}{cccc}
0 & 0 & m_{1} & m_{2} \\
0 & 0 & m_{2} & m_{1} \\
m_{1} & m_{2} & M_{1} & M_{2} \\
m_{2} & m_{1} & M_{2} & M_{1}
\end{array}\right)
$$

The mass matrix can be simplified by changing to the parity diagonal basis $\nu_{L}^{ \pm}=\frac{\nu_{L} \pm\left(N_{R}\right)^{c}}{\sqrt{2}}$, and $\nu_{R}^{ \pm}=\frac{\nu_{R} \pm\left(N_{L}\right)^{c}}{\sqrt{2}}$. In this basis the mass matrix has the form

$$
\mathcal{M}=\left(\begin{array}{cccc}
0 & 0 & 0 & m_{+} \\
0 & 0 & m_{-} & 0 \\
0 & m_{-} & M_{-} & 0 \\
m_{+} & 0 & 0 & M_{+}
\end{array}\right)
$$

where $m_{ \pm}=m_{1} \pm m_{2}$ and $M_{ \pm}=M_{1} \pm M_{2}$. The mass matrix can now be easily diagonalised because it is essentially two copies of the $2 \times 2$ mass matrix Eq.(18). In the limit $M_{ \pm} \gg m_{ \pm}$, the mass matrix Eq.(24) has eigenvalues:

$$
m_{+}^{2} / M_{+}, m_{-}^{2} / M_{-}, M_{+}, M_{-},
$$

and eigenvectors (which are the mass eigenstates):

$$
\nu_{1}=\nu_{L}^{+}, \nu_{2}=\nu_{L}^{-}, \nu_{3}=\nu_{R}^{+}, \nu_{4}=\nu_{R}^{-} .
$$

Thus, in the one generation case, there is effectively only two state mixing:

$$
\begin{aligned}
\nu_{L} & =\frac{\nu_{L}^{+}+\nu_{L}^{-}}{\sqrt{2}} \\
& =\frac{\nu_{1}+\nu_{2}}{\sqrt{2}} .
\end{aligned}
$$


Thus in this case the averaged neutrino oscillation probability function $\mathcal{P}$ (defined in Eq.(13)) is 1/2. In the physical case of three generations, in general $\nu_{L}^{+}$and $\nu_{L}^{-}$will each be linear combinations of three mass eigenstates:

$$
\nu_{e L}=\frac{\alpha_{1} \nu_{1}^{\prime}+\alpha_{2} \nu_{2}^{\prime}+\alpha_{3} \nu_{3}^{\prime}}{\sqrt{2}}+\frac{\beta_{1} \nu_{1}^{\prime \prime}+\beta_{2} \nu_{2}^{\prime \prime}+\beta_{3} \nu_{3}^{\prime \prime}}{\sqrt{2}},
$$

where $\alpha_{1}^{2}+\alpha_{2}^{2}+\alpha_{3}^{2}=\beta_{1}^{2}+\beta_{2}^{2}+\beta_{3}^{2}=1$ and $\nu_{i}^{\prime}, \nu_{i}^{\prime \prime}$ are the six light physical mass eigenstates. In this case, the effect of the neutrino oscillations averaged over many oscillations is to reduce the electron neutrino flux by a factor $\mathcal{P}$ where

$$
1 / 6 \leq \mathcal{P} \leq 1 / 2 .
$$

To determine the precise value of $\mathcal{P}$ one must know the actual values of the parameters $\alpha_{i}, \beta_{i}$ which can only be determined if the neutrino mass matrix is known. Note however that if intergenerational mixing follows the pattern suggested by the quarks (i.e. small intergenerational mixing angles) then one would expect that the one generation result should be a good approximation and that $\mathcal{P}$ should be nearly $1 / 2$.

Our second example is also a version of the see-saw model which involves adding only three gauge singlets $N_{i R}$ (rather than six as in the previous example above) which are parity diagonal (i.e. under the parity transformation $\left.N_{i R} \leftrightarrow \gamma_{0}\left(N_{i R}\right)^{c}\right)$. In this case, for one generation, we have the following mass terms (assuming the minimal Higgs sector Eq.(6)):

$$
\mathcal{L}_{\text {mass }}=m\left(\bar{\nu}_{L} N_{R}+\bar{\nu}_{R}\left(N_{R}\right)^{c}\right)+M \bar{N}_{R}\left(N_{R}\right)^{c}+\text { H.c. }
$$

where the parity transformation (Eq.(5)) interchanges $\nu_{L} \leftrightarrow \gamma_{0} \nu_{R}$ and $N_{R} \leftrightarrow$ $\gamma_{0}\left(N_{R}\right)^{c}$ (note that, as before $\nu_{L}\left(\nu_{R}\right)$ is an ordinary neutrino (mirror neutrino) and is the $S U(2)_{1}\left(S U(2)_{2}\right)$ partner of the electron (mirror electron).) In this model, for the simplified case of one generation, the phases of the fields can be chosen so that all of the masses are real. Thus the reality assumption of the neutrino mass matrix is redundant unless there is intergenerational mixing. If intergenerational mixing is small, which is possible (c.f case of quarks), then the simplified case of one generation will be a good approximation to the physical case of three generations. If we change to the parity diagonal basis, by defining $\nu_{ \pm}=\frac{\nu_{L} \pm\left(\nu_{R}\right)^{c}}{\sqrt{2}}$, then the mass matrix has 
the form:

$$
\mathcal{L}_{\text {mass }}=\left(\bar{\nu}_{-L}, \bar{\nu}_{+L},\left(\bar{N}_{R}\right)^{c}\right)\left(\begin{array}{ccc}
0 & 0 & 0 \\
0 & 0 & m \\
0 & m & M
\end{array}\right)\left(\begin{array}{c}
\left(\nu_{-L}\right)^{c} \\
\left(\nu_{+L}\right)^{c} \\
N_{R}
\end{array}\right)+H . c . .
$$

Assuming that $M \gg m$, then there are 3 Majorana mass eigenstates $N_{R}$, $\nu_{+L}$ and $\nu_{-L}$ with eigenvalues $M, m^{2} / M, 0$. In this one generation case, there

is effectively two-state mixing, $\nu_{e L}=\nu_{+L}+\nu_{-L} / \sqrt{2}$ and $\mathcal{P}=1 / 2$. For the physical case of three generations, in general $1 / 6 \leq \mathcal{P} \leq 1 / 2$ (assuming that $M_{i} \gg m_{j}$ ), however as before, if intergenerational mixing is suppressed then we would expect $\mathcal{P}$ to be close to $1 / 2$.

\section{Atmospheric neutrino anomaly and concluding re- marks}

It may be possible to explain the atmospheric neutrino anomaly (Eq.(2)) if the muon neutrino oscillates into the mirror neutrino on its way down from the atmosphere. The oscillation length for the electron neutrino can be large enough so that it does not have time to oscillate (but short enough so that the solar neutrinos oscillate!). Obviously, since the atmosphere is not very deep there will not be a large range of parameters in which the muon neutrino will have time to oscillate (this is unlike the case for solar neutrino oscillations). Another problem with this solution is that it does not seem to be consistent with upcoming muon events coming from muon neutrinos travelling through the Earth. However this conclusion relies heavily on the absolute neutrino flux estimate $[6,13]$. It may be possible that the absolute muon neutrino flux has been over estimated, and that the atmospheric neutrino anomaly is an indication that the muon neutrinos mix nearly maximally with a mirror neutrino (which is effectively sterile with regard to ordinary matter). Of course this possibility is very speculative at the moment, and we await further neutrino experiments with interest to see whether the atmospheric neutrino anomaly is stable (i.e. time independent).

To summarise, we have examined neutrino oscillations in the exact parity model. We have shown that the effect of vacuum neutrino oscillations can be large when neutrinos mix with their mirror partners. We proved some general results which are nearly independent of the details of the neutrino 
mass matrix. In particular we examined the possibility of massive neutrinos and we showed (in the context of exact parity symmetry) that if the neutrino mass matrix is real, then the neutrino flux of a source of weak eigenstate neutrinos (such as the sun) is reduced by a factor greater than 2 provided the neutrino oscillation lengths are small enough (i.e. neutrino mass differences are big enough) compared with the size of the source (the sun) or detector (the Earth). The sun is very big, so there is quite a large range of parameters (about 8 orders of magnitude) in which the flux of electron neutrinos as measured in the experiments would be reduced by a factor of 2 or more. Thus, we argue that if the solar neutrino deficit and/or atmospheric neutrino anomalies are signals of some new physics, then this new physics might be vacuum neutrino oscillations. They can be naturally large if parity is a exact symmetry of nature.

\section{Acknowledgements}

I would like to thank Henry Lew and Ray Volkas for many discussions concerning exact parity.

\section{References}

[1] R. Davis, in Proceedings of the 21st International cosmic ray conference, ed. R. J. Protheroe (University of Adelaide Press, Australia, 1990) Vol.2 p 143; Kamiokande collabroration, K. S. Hirata et al, Phys. Rev. Lett. 65, 1297 (1990); Phys. Rev. D44, 2241 (1991); GALLEX Collaboration, P. Auselmann et al; Phys. Lett. B285, 376 (1992); GALLEX Collaboration, presented by F. van Feilitzsch, in the Proceedings of the International conference "Neutral currents twenty years later", Paris 1993, to appear; SAGE Collaboration, A. I. Abazov et al, Phys. Rev. Lett. 67, 3332 (1991).

[2] J. N. Bachall et al, Rev. Mod. Phys. 54, 767 (1982); J. N. Bahcall and R. K. Ulrich, Rev. Mod. Phys. 69, 297 (1988); J. N. Bahcall and M. H. Pinsonneault, Rev. Mod. Phys. 64, 885 (1992). 
[3] S. Turck-Chieze et al, Ap. J. 335, 415 (1988).

[4] D. R. O. Morrison, Int. J. of Mod. Phys. D1, 281 (1992); CERN preprint/93-98 (1993).

[5] Kamiokande Collaboration, K. S. Hirata et al, Phys. Lett. B280, 146 (1992); IMB Collaboration, D. Casper et al, Phys. Rev. Lett. 66, 2561 (1991); R. Becher-Szendy et al., Phys. Rev. D46, 3720 (1992).

[6] T. K. Gaisser, T. Stanev and G. Barr, Phys. Rev. D38, 85 (1988); G. Barr, T. K. Gaisser and T. Stanev, Phys. Rev. D39, 3532 (1989).

[7] B. Pontecorvo, Sov. JETP 26, 984 (1968); V. Gribov and B. Pontecorvo, Phys. Lett. B28, 493 (1969).

[8] L. Wolfenstein, Phys. Rev. D17, 2369 (1978); S. P. Mikheyev and A. Yu. Smirnov, Nuovo. Cimento 9C, 17 (1986).

[9] R. Foot, H. Lew and R. R. Volkas, Mod. Phys. Lett. A7, 2567 (1992).

[10] R. Foot, H. Lew and R. R. Volkas, Phys. Lett. B272, 67 (1991). The idea that parity can be a symmetry of nature was discussed earlier by T. D. Lee and C. N. Yang, Phys. Rev. 104, 254 (1956); A. Salam, Nuovo Cimento, 5, 299 (1957); V. Kobzarev, L. Okun and I. Pomeranchuk, Sov. J. Nucl. Phys. 3, 837 (1966); M. Pavsic, Int. J. Theor. Phys. 9, 229 (1974); See also Ref [11].

[11] S. L. Glashow, Phys. Lett. B167, 35 (1986); E. D. Carlson and S. L. Glashow, Phys. Lett. B193, 168 (1987); see also B. Holdom, Phys. Lett. B166, 196 (1985).

[12] Particle data group, Phys. Rev. D (1992).

[13] M. Honda et al, Phys. Lett. B248, 193 (1990); H. Lee and Y. S. Koh, Nuovo Cim. 105B, 883 (1990); D. H. Perkins, Nucl. Phys. B399, 3 (1993). 